\title{
Severe Bladder Outlet Obstruction in a 7-Week-Old Infant Presenting with Features of Prune Belly Syndrome: A Case Report
}

\author{
Muhammad Sule Baba, Sadisu Mohammed Ma'aji, Sule Ahmed Sa'idu, \\ Amina Bello Muhammad Shamaki, Usman Aminu Umar
}

Department of Radiology, Usmanu Danfodiyo University, Sokoto, Nigeria

\begin{abstract}
Prune Belly syndrome is characterized by urinary tract anomalies, deficient abdominal muscle and bilateral cryptorchidism. It has an incidence of about 1 in 50,000 live births with male preponderance in $95 \%$. This is a 7 weeks old male infant that was referred from a peripheral health care centre for a micturating cystourethrography on account of difficulty in micturition, distended abdomen and restlessness since birth. The patient had an abdominal ultrasonography that showed bilateral severe hydronephrosis, megaureters and thick-walled urinary bladder. A micturating cystourethrogram showed pine shaped urinary bladder with bilateral vesicoureteric reflux more marked on the right. Plain abdominal radiograph showed markedly distended abdomen and gas distended bowel loops with thinning of anterior abdominal wall muscles. The referring physician was advised to make a prompt referral of this case to a higher centre for better management. We report the radiologic findings of this case presenting with features of Prune Belly syndrome.
\end{abstract}

Key words: Cryptorchidism, cystitis, hydronephrosis, megaureter

\section{INTRODUCTION}

Prune belly syndrome (PBS) also known as Eagle-Barrett syndrome is a rare congenital disorder affecting about 1 in 30,000 live births with a male preponderance in about $96 \%$ of affected individuals. ${ }^{[1-3]}$

PBS has no exact etiology, however, it is believed that there is some genetic inheritance and possible association with trisomy 18 and 21. ${ }^{[1,3,4]}$ Most cases are sporadic, however, rarely familial cases have been described. ${ }^{[5]}$

PBS also called triad syndrome is characterized by a triad of findings which include deficient abdominal

\begin{tabular}{|l|l|}
\hline \multicolumn{2}{|c|}{ Access this article online } \\
\hline Publisher & \multirow{2}{*}{$\begin{array}{l}\text { Website: } \\
\text { www.ijdms.in }\end{array}$} \\
\hline & DOI: 10.30954/IJDMS.1.2020.12 \\
\hline
\end{tabular}

wall musculatures, bilateral cryptorchidism, and urinary tract abnormalities; some of which are bilateral severe hydronephrosis, megaureter, and megacystis. ${ }^{[1,3,5,6]}$

The anterior abdominal wall muscles are deficient in about $30 \%$ of cases and often replaced by dense collagenous tissue and hypoplastic with areas of absence in about $70 \%{ }^{[5,7]}$ The skin of the anterior abdominal wall is wrinkled, thinned out, and laxed appearing like a prune, hence, the name of the syndrome..$^{[1,5]}$

Cryptorchidism occurs in about $95 \%$ of cases, excluding the female patients, however, all presented with urethral atresia, increasing the interest that urethral obstructions are the underlying cause of PBS. ${ }^{[5,7]}$

In patients with PBS, the anomalies of the genitourinary system mainly consist of renal dysplasia and hypoplasia, ureteral dilatation, megacystis, dilated prostatic urethra, and prostatic hypoplasia. ${ }^{[1,5,6]}$ The vesicoureteric junction is usually markedly patent with associated vesicoureteric reflux occurring in about $75 \%$ of cases. ${ }^{[1,5,6]}$

\footnotetext{
Address for Correspondence:

Dr Muhammad Sule Baba, Department of Radiology, Usmanu Danfodiyo University, Sokoto, Nigeria.

E-mail: muhammadsule@yahoo.com
} 
Patients with PBS also show an enlarged urinary bladder that is often thickened and non-trabeculated, with a quarter and occasionally half of the cases presenting with either a patent urachus or its diverticulum. ${ }^{[5]}$ Urethral obstruction is equally an important finding seen in about $0-100 \%$ of subjects with PBS. ${ }^{[5]}$

PBS patients may also present with associated nongenitourinary conditions which may include cardiopulmonary, gastrointestinal, and musculoskeletal anomalies. ${ }^{[8-11]}$

PBS in the neonates has been categorized into three groups by Woodard ${ }^{[12]}$ based on presentation; Category I has patients with severe oligohydramnios secondary to renal dysplasia with or without outlet obstruction resulting to severe pulmonary hypoplasia and skeletal abnormalities, ${ }^{[13]}$ they have a severe form of the syndrome with a poor outcome. Category II of PBS experiences moderate renal insufficiency and moderate-severe hydroureteronephrosis, pulmonary hypoplasia is often not a prominent feature. Category III consists of patients with mild triad features or incomplete forms with normal or impaired renal functions and there is no associated pulmonary insufficiency. ${ }^{[8]}$

\section{CASE REPORT}

This is a 7-week-old full-term male infant that presented to the radiology department from a peripheral health center in a neighboring state on account of distended abdomen, respiratory distress, difficult and painful micturition, and generalized weakness of the limbs since birth.

The mother has had eight deliveries with no adequate antenatal hospital care, she denied a history of similar occurring's in the other siblings but accepted intake of local herbs during the index pregnancy on different occasions following febrile illnesses.

The child appeared to be in the form of distress with distended abdomen having a wrinkled appearance and visible peristaltic activities of the bowel loops, has prominence of the lumbar regions and suprapubic region, he is not pale, not dehydrated but has low-grade muscle tone (Grades I-II) of all the limbs.

The patient had an abdominal and pelvic ultrasonography with plain radiography and micturating cystourethrography (MCUG).

The MCUG showed a distended urinary bladder having a pine tree appearance with dilated prostatic urethra that abruptly terminated with a distal beaking. The membranous and anterior urethras were, however, not demonstrated despite all maneuvers. There is associated bilateral vesicoureteric reflux more marked on the right where marked dilatation and tortuosity of the ureter were demonstrated [Figures 1-3].

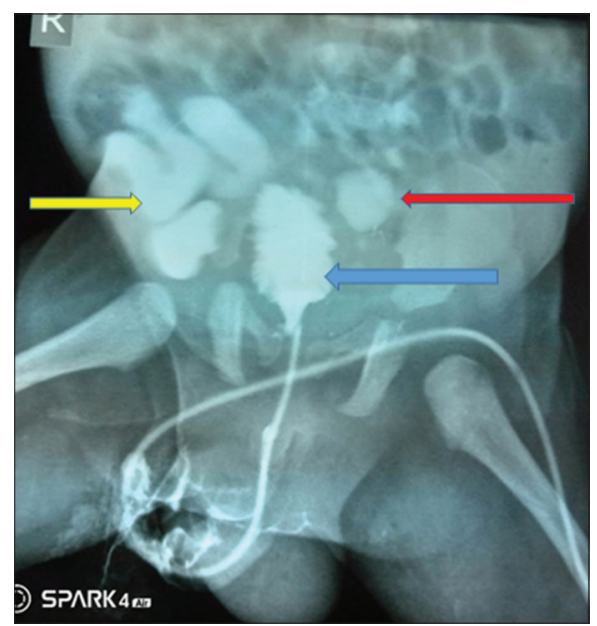

Figure 1: Micturating cystourethrography showing a pine tree appearance of the urinary bladder (left blue arrow) with bilateral dilated ureters; vesicoureteric reflux; moderate on the left (red left arrow) while more marked on the right where tortuosity of the ureter is noted (yellow right arrow)

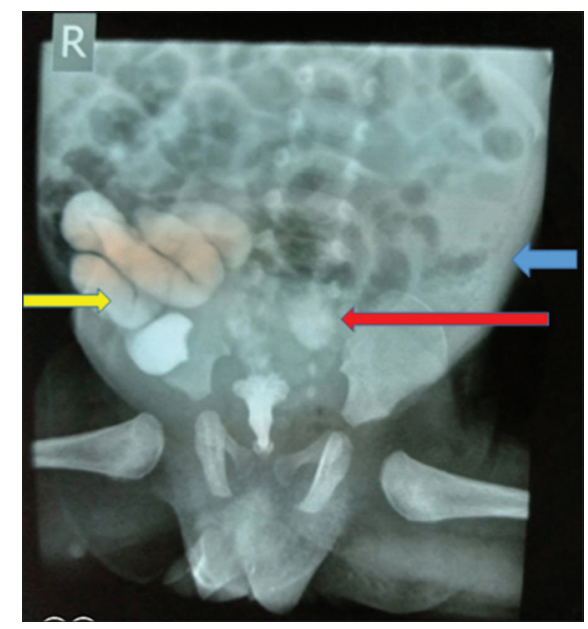

Figure 2: An micturating cystourethrography following removal of the catheter and subsequent draining of the contrast medium from the urinary bladder; the urinary bladder appear collapsed with bilateral vesicoureteric reflux more marked on the right (right yellow and left red arrow). Distended abdomen with bilateral flank fullness (left flank fullness; left blue arrow) and gas distended bowel loops also demonstrated

Thinning of the soft-tissue shadows in the region of the anterior abdominal wall cranially most likely from hypoplasia [Figure 3].

The plain abdominal radiograph showed a markedly distended abdomen with bilateral fullness of both flanks, marked gas distended bowel loops with the demonstrated skeletal system appearing within normal limits excluding any form of anomalies [Figure 4].

The abdominopelvic ultrasound showed normal abdominal situs of all organs with marked enlargement of both kidneys (right $=12 \mathrm{~cm}$ and left $=12.5 \mathrm{~cm}$, respectively) with 


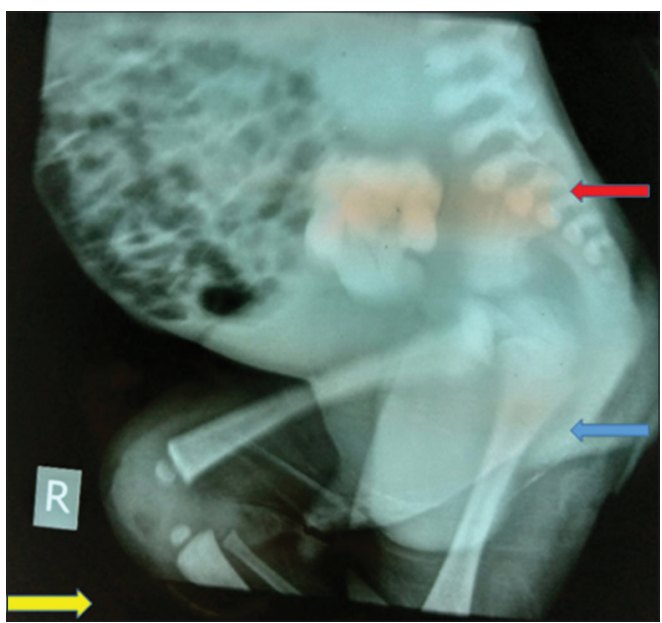

Figure 3: An micturating cystourethrography right lateral projection showing a dilated and tortuous right ureter (left blue arrow) with thinning of the soft tissue outline in the anterior abdominal wall more marked superiorly (right yellow arrow). Normal lumbosacral spine was also demonstrated (left red arrow)

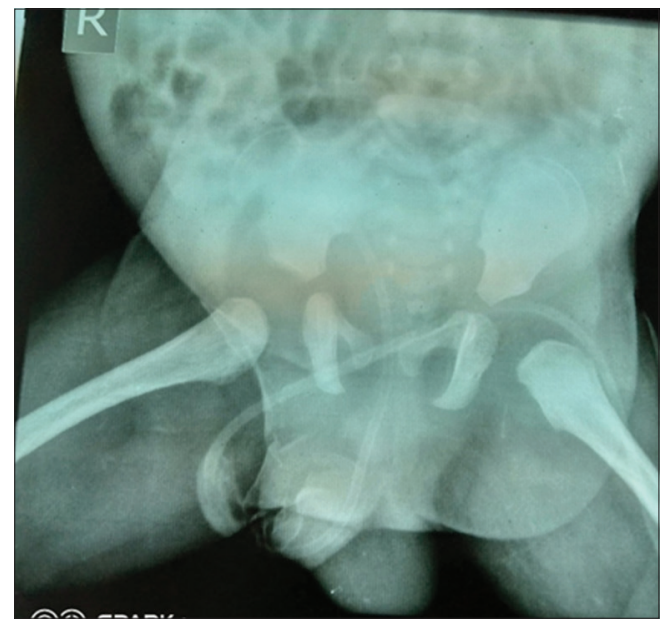

Figure 4: Plain radiograph anterior-posterior view showing the pelvis and lower abdomen; normal bones were demonstrated with gas distended bowel loops and bilateral fullness of the flanks

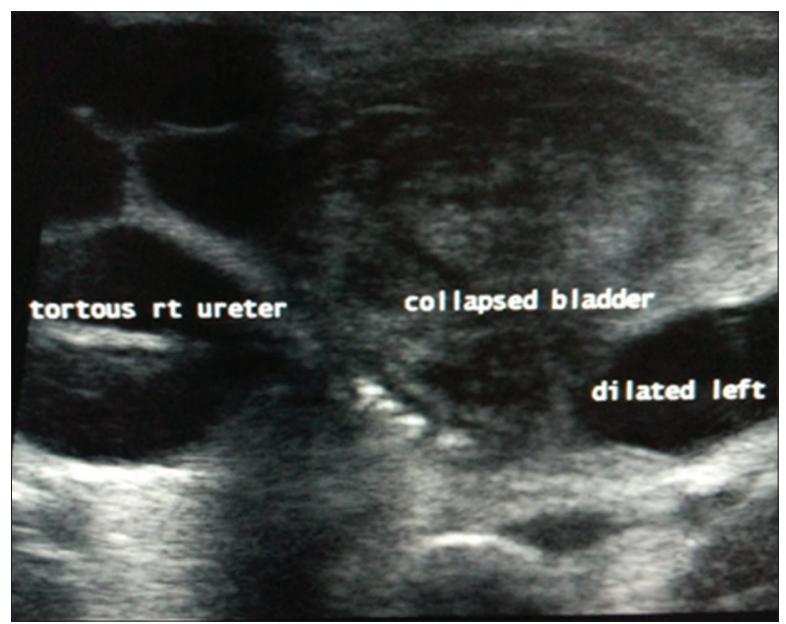

Figure 5: An ultrasonogram of the emptied urinary bladder appearing as a bulky structure with dilated distal ureters more marked on the right associated severe hydroureteronephrosis more marked on the right where tortuosity of the distal aspect was also demonstrated [Figure 5]. The urinary bladder appeared enlarged and showed marked wall thickening $(28 \mathrm{~mm})$ with significant volume of urine having suspended echoes most likely megacystis; the bladder also appeared bulk like after emptying its content by a feeding tube (smallest size of Foley's catheter was unable to pass through the urethra).

Bilateral absence of the testicles within the hemiscrotal sacs in keeping with cryptorchidism was also demonstrated following a testicular ultrasonography.

The findings of severe bladder outlet obstruction with megaureters and megacystis, cryptorchidism, and marked thinning (hypoplastic) of the anterior abdominal wall with associated marked gas distended bowel loops prompted the diagnosis of PBS in this patient.

The findings were communicated to the referring physician with the advice of referring the patient to a higher medical center where adequate management of this patient can be achieved.

\section{DISCUSSION}

PBS is a rare congenital disorder affecting about 1 in 30,000 live births with a male preponderance in about $96 \%$ of affected individuals. ${ }^{[1-3]}$ The index case happens to be a male, thereby conforming to these literatures.

PBS also called triad syndrome is characterized by a triad of findings which include deficient abdominal wall musculatures, bilateral cryptorchidism, and urinary tract abnormalities; some of which are bilateral severe hydronephrosis, megaureter, and megacystis. ${ }^{[1,3,5,6]}$ The index case also had these features which were bilateral cryptorchidism, bilateral severe renomegaly, severe hydroureteronephrosis, megaureters, and megacystis with a thinned and deficient anterior abdominal wall musculature, thereby conforming to these literatures.

The anterior abdominal wall muscles are deficient in about $30 \%$ of cases, cryptorchidism occurs in about $95 \%$ of cases and associated vesicoureteric reflux also occurring in about $75 \%$ of cases. ${ }^{[1,5-7]}$ The patient under review also had these documented features, thereby conforming to that reported in these literatures.

On physical examination of patients with PBS, the classical features of distended abdomen with visible bowel peristaltic activities, weakness and hypotonia of the limbs, respiratory distress, and prominent suprapubic and flank/ lumbar regions are some of the findings as documented 
in most literatures, the index case also had most of these features, thereby conforming to that documented in the literature.

PBS has no exact etiology, however, it is believed that there is some genetic inheritance and possible association with trisomy 18 and $21{ }^{[1,3,4]}$ The index case had no known cause of the syndrome and no history of similar occurrence among siblings, he also had no feature of trisomy 18 and 21 , thereby agreeing to these literatures.

PBS patients may also present with associated nongenitourinary conditions which may include cardiopulmonary, gastrointestinal, and musculoskeletal anomalies. ${ }^{[8-11]}$ The patient had no documented cardiopulmonary and skeletal anomalies but presented with marked gaseous distended bowel loops.

PBS in the neonates has been categorized into three groups by Woodard ${ }^{[12]}$ based on presentation, the index case most likely belongs to Category II of PBS; the patients experience moderate renal insufficiency and moderatesevere hydroureteronephrosis, and pulmonary hypoplasia is often not a prominent feature. The index case has no feature of pulmonary hypoplasia but has severe bilateral hydroureteronephrosis agreeing to this literature.

Most cases of PBS are often diagnosed using ultrasonography, plain abdominal radiography with $\mathrm{MCUG},{ }^{[1-13]}$ this index case also had plain abdominal radiography and abdominopelvic ultrasonography with an MCUG, these demonstrated most of the features of PBS, thereby agreeing to these literatures.

The management of cases of PBS is often multidisciplinary, tasking and often systematic in approach to achieve the best and improve the patients quality of life among the few cases that often survive after delivery; the index case was referred only for MCUG, on which he was referred back with the findings of the examinations. The referring physician was, however, advised to refer the index case to a higher medical center where he would be given the desired management.

\section{CONCLUSION}

Severe bladder outlet obstruction with or without features of PBS can be life threatening and often leading to nephropathy with subsequent pulmonary hypoplasia and patient demise, prompt diagnosis following an MCUG, abdominopelvic ultrasonography, and radiography should be encouraged to save the lives of these patients.

\section{REFERENCES}

1. Suni KS, Setu R. Prune belly syndrome: A rare case report. J Nat Sci Biol Med 2005;8:255-7.

2. Baird PA, MacDonald BC. An epidemiologic study of congenital malformations of the anterior abdominal wall in more than half a million consecutive live births. Am J Hum Genet 1981;33:470-8.

3. Tagorw KR, Ramineni AK, Lakshmi AR, Bhavani N. Prune belly syndrome. Case Rep Pediatr 2011;2011:121736.

4. Ramasamy R, Haviland M, Woodard JR, Barone JG. Patterns of inheritance in familial prune belly syndrome. Urology 2005;65:1227.

5. Herman TE, Siegel MJ. Imaging case book prune belly syndrome. J Perinat 2009;29:69-71.

6. Wheatley JM, Stephens FD, Hutson JM. Prune belly syndrome: Ongoing controversies regarding pathogenesis and management. Semin Pediatr Surg 1996;5:95-106.

7. Reinberg Y, Shapiro E, Manivel JC, Manley CB, Pettinato G, Gonnzalez R. Prune belly syndrome in females: A triad of abdominal musculature deficiency and anomalies of the urinary and genital system. J Pediatr 1991;118:395-8.

8. Arlem AM, Nawaf C, Kirsch AJ. Prune belly syndrome: Current perspectives. Pediatric Health Med Ther 2019;10:75-81.

9. Hassett S, Smith GH, Holland AJ. Prune belly syndrome. Pediatr Surg Int 2012;28:219-28.

10. Smolkin T, Soudack M, Goldstein I, Sujov P, Makhoul IR. Prune belly syndrome: Expanding the phenotype. Clin Dysmorphol 2008;17:133-5.

11. Seidel NE, Arlen AM, Smith EA, Kirsch AJ. Clinical manifestation and management of prune-belly syndrome in a large contemporary pediatric population. Urology 2015;85:211-5.

12. Woodard JR. The prune belly syndrome. Urol Clin North Am 1978;5:75-93.

13. Woods AG, Brandon DH. Prune belly syndrome: A focused physical assessment. Adv Neonatal Care 2007;7:132-43.

How to cite this article: Baba MS, Ma'aji SM, Sa'idu SA, Shamaki ABM, Umar UA. Severe Bladder Outlet Obstruction in a 7-Week-Old Infant Presenting with Features of Prune Belly Syndrome: A Case Report. Int J Dent Med Spec 2020;7(1):49-52.

Source of Support: None; Conflicts of Interest: None 